\title{
The impact of restaurant consumption among US adults: effects on energy and nutrient intakes
}

\author{
Binh T Nguyen ${ }^{1, *}$ and Lisa M Powell ${ }^{2}$ \\ ${ }^{1}$ Economic and Health Policy Research, American Cancer Society, Inc., 250 Williams Street, Atlanta, GA 30303, \\ USA: ${ }^{2}$ Health Policy and Administration, School of Public Health, University of Illinois at Chicago, Chicago, IL, USA
}

Submitted 21 November 2013: Final revision received 25 March 2014: Accepted 6 May 2014: First published online 30 July 2014

\begin{abstract}
Objective: To examine the effect of fast-food and full-service restaurant consumption on adults' energy intake and dietary indicators.

Design: Individual-level fixed-effects regression model estimation based on two different days of dietary intake data was used.

Setting: Parallel to the rising obesity epidemic in the USA, there has been a marked upward trend in total energy intake derived from food away from home.

Subjects: The full sample included 12528 respondents aged 20-64 years who completed $24 \mathrm{~h}$ dietary recall interviews for both day 1 and day 2 in the National Health and Nutrition Examination Survey (NHANES) 2003-2004, 2005-2006, 2007-2008 and 2009-2010.

Results: Fast-food and full-service restaurant consumption, respectively, was associated with an increase in daily total energy intake of $813.75 \mathrm{~kJ}$ (194.49 kcal) and $858.04 \mathrm{~kJ}(205.21 \mathrm{kcal})$ and with higher intakes of saturated fat $(3.48 \mathrm{~g}$ and $2.52 \mathrm{~g}$ ) and $\mathrm{Na}(296.38 \mathrm{mg}$ and $451.06 \mathrm{mg})$. Individual characteristics moderated the impacts of restaurant food consumption with adverse impacts on net energy intake being larger for black adults compared with their white and Hispanic counterparts and greater for middle-income $v$. high-income adults.

Conclusions: Adults' fast-food and full-service restaurant consumption was associated with higher daily total energy intake and poorer dietary indicators.
\end{abstract}

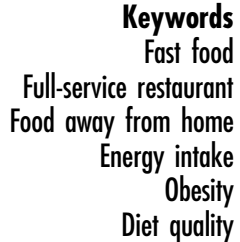

The USA is one of the most obese nations in the world, with the age-adjusted prevalence of obesity at $35.5 \%$ among adult men and $35.8 \%$ among adult women in $2009-2010^{(1)}$. With obesity linked to diabetes, heart disease, stroke and some cancers $^{(2)}$, health-care spending attributed to obesity reached \$US 147 billion in 2008, and the costs are projected to increase dramatically in the future ${ }^{(3)}$. Parallel to the rising obesity epidemic, there has been a marked upward trend in total energy intake derived from food away from home (FAFH). In 1977-1978, the contribution of intake from fastfood and full-service restaurants to total energy intake was $14.2 \%$ for young adults (aged 19-39 years) and 11.5\% for older adults (aged 40-59 years) ${ }^{(4)}$. In 2007-2008, the contribution of restaurant energy to total energy intake increased to $24 \%$ (13\% for fast-food and $11 \%$ for full-service restaurants) for adults aged 20-64 years ${ }^{(5)}$. In addition, in $2007-2008$, on a given day, $36 \%$ and $27 \%$ of US adults aged 20-64 years consumed foods and beverages from fast-food and full-service restaurant sources, respectively ${ }^{(5)}$. Moreover, FAFH as a share of food expenditures increased gradually from $30 \%$ in 1977 to $43 \%$ in 2012, although its share stagnated and declined slightly to about $42 \%$ in the recent recession $(2007-2009)^{(6)}$.
A number of studies have found an association between FAFH consumption and greater total energy intake and poorer nutrient intakes among adults ${ }^{(7-11)}$. Adults who ate fast food had higher intakes of energy, total fat, saturated fat and $\mathrm{Na}$, and lower intakes of vitamins, milk, fruits and vegetables than those who did not report eating fast food $^{(7-9,12)}$. However, studies often focused on fast-food but not full-service restaurant consumption with the exception of one recent paper that found similar impacts of fast-food and full-service food consumption for children ${ }^{(13)}$. Moreover, some studies using cross-sectional or adjusted mean comparison analysis ${ }^{(8,9)}$ often missed two important potential sources of endogeneity of consumer preferences: (i) those who eat larger or less healthy meals may prefer to eat at restaurants; and (ii) individuals might offset kilojoules eaten at restaurants by reducing energy intake at other times during the day. Thus, the positive correlation between restaurant consumption and energy intake and diet quality may be confounded by a variety of unobserved individual-level characteristics and preferences. Moreover, the differential effect of restaurant consumption on different sub-populations such as blacks compared with whites and Hispanics, low income 
compared with high income, and younger compared with older adults has not been extensively studied, although it is well known that their rates of obesity are different ${ }^{(14)}$.

The present study aimed to examine the relationship between fast-food and full-service restaurant consumption and energy and nutrient intakes for adults aged 20-64 years in the USA. To control for individuals' unobserved characteristics, we estimated multivariate individual-level fixedeffects regression models. Such models have previously been estimated to examine the relationship between FAFH and restaurant consumption on energy intake and diet quality $^{(7,13,15)}$. Our adult sample is drawn from the nationally representative dietary recall data of the National Health and Nutrition Examination Survey (NHANES) 2003-2010. Our research focused on the effects of restaurant consumption on total energy intake and other key elements of which excessive consumption might be harmful, including saturated fat, salt, sugar and $\mathrm{Na}$ intakes ${ }^{(16-18)}$. We assessed the differential effects of restaurant consumption by gender, ethnicity and income sub-populations as well as by meal occasion. We also examined racial and socio-economic status differences by age group and by gender.

\section{Methods}

\section{Data}

The dietary recall data from the nationally representative NHANES 2003-2004, 2005-2006, 2007-2008 and 2009-2010 waves were used. A complete description of the data collection procedures and survey design is given elsewhere. ${ }^{(19)}$ Non-pregnant individuals aged 20-64 years who completed two dietary interviews were included in our sample. Subgroup analyses by gender, race/ethnicity, income and age were conducted. Because of the relatively small sample size for the other race category, racial/ethnic subgroup analysis was focused on non-Hispanic whites, non-Hispanic blacks and Hispanics. Low-income adults were defined as individuals in families with income $<130 \%$ of the federal poverty level (FPL), middle-income adults as $\geq 130 \%$ and $<300 \%$ of the FPL, and high-income adults as $\geq 300 \%$ of the FPL. There were two subgroups based on age: young adults aged 20-34 years and older adults aged 35-64 years.

The NHANES included two non-consecutive $24 \mathrm{~h}$ dietary recalls for which respondents reported all foods and beverages consumed in the prior $24 \mathrm{~h}$. The US Department of Agriculture's Automated Multiple-Pass Method was used for data collection. Day 1 interviews were conducted by trained dietary interviewers in a mobile examination centre and day 2 interviews were collected by telephone 3 to $10 \mathrm{~d}$ later. Over the sample period from 2003-2004 through $2009-2010,85.5 \%$ of dietary recalls from day 1 had day 2 dietary recall data available. Our full sample included 12528 respondents who completed $24 \mathrm{~h}$ dietary recall interviews for both day 1 and day 2 in the NHANES from 2003-2004 to 2009-2010.
The survey respondents were asked about the source of each food and beverage item in terms of where it was obtained (e.g. from a store, fast-food restaurant, full-service restaurant, etc.). From this question, we constructed the following measures as dichotomous indicators of whether the individual consumed food or beverages from: (i) fastfood restaurants and (ii) full-service restaurants. Moreover, non-restaurant FAFH included all food and beverage items consumed away from home that were not sourced from a fast-food or full-service restaurant. Our analyses also controlled for whether the food or beverage items were consumed on a weekday or weekend day. Based on the reported quantity of all food and beverage items consumed, the energy and nutritional contents of each item were coded in the NHANES according to US Department of Agriculture databases ${ }^{(19,20)}$.

\section{Study design and statistical analyses}

We specified an individual-level fixed-effects regression model where each individual serves as his/her own control, based on the two different days of intake data. This model removed the effects of all standard observed characteristics fixed over time such as age, gender and race, as well as the unobserved characteristics such as preferences for particular foods that affect both the incidence and food choices when eating away from home. Moreover, given the short time span between the $24 \mathrm{~h}$ dietary recalls of day 1 and day 2 , it also removed the effects of characteristics such as marital status, education and income.

The dependent variables included the change in reported total energy intake (kilojoules/kilocalories), sugar (grams), saturated fat (grams) and $\mathrm{Na}$ (milligrams) throughout the surveyed day (day 1-day 2). The key independent variables of the models were whether or not any food or beverages consumed came from a fast-food or a full-service restaurant in the $24 \mathrm{~h}$ period. The corresponding coefficients, therefore, illustrated the change in total energy intake (sugar, saturated fat, $\mathrm{Na}$, etc.) associated with fast-food or fullservice restaurant food consumption. We also controlled for time-varying covariates that may be correlated with daily energy intake such as whether the intake day was a weekday $v$. a weekend day ${ }^{(21)}$ and whether any consumption that day was eaten from a non-restaurant away-fromhome source. We controlled for whether an individual ate away from home on day $1 v$. day 2 due to the difference in interview methods across days. In addition, we used a second fixed-effects model specification to estimate the effect of different fast-food and full-service restaurant meal occasions (breakfast, lunch, dinner and snack) on change in total energy intake. We restricted our estimation to adults aged 20-64 years and excluded older adults aged 65 years and above to maximize the comparability of dietary intake patterns. We undertook analyses separately by age, gender, race/ethnicity and household income subgroups. We also conducted analyses by age and gender further sub-stratified 
by race and income subgroups. We performed statistical testing to compare differences in regression coefficient estimates across subgroups.

The statistical software package Stata 11.1 (2009) was used for all estimation. The estimation accounted for the NHANES complex, multistage probability sampling design to provide representative samples of adults aged 20-64 years. The study was approved by the Institutional Review Board of the University of Illinois at Chicago.

\section{Results}

The prevalence of fast-food restaurant, full-service restaurant and non-restaurant FAFH food consumption and total energy and nutrient intakes for the full sample are presented in Table 1 . On day $1,35 \%$ of our sample reported consumption from fast-food sources, $28 \%$ reported eating full-service restaurant food and $61 \%$ reported eating non-restaurant FAFH. On average, total energy intake was $9548 \mathrm{~kJ}(2282 \mathrm{kcal})$ on day 1 and $8866 \mathrm{~kJ}$ (2119 kcal) on day 2.

Table 2 presents the regression results examining whether fast-food and full-service restaurant food consumption was related to energy and nutrient intakes for the full sample as well as the age and gender subgroups. Fast-food and full-service restaurant consumption was associated with a net increase in daily total energy intake of $813.75 \mathrm{~kJ}$ $(194.49 \mathrm{kcal})$ and $858.04 \mathrm{~kJ}(205.21 \mathrm{kcal})$, respectively. The additional kilojoules from restaurant consumption were equivalent to almost $10 \%$ of total daily energy intake. Furthermore, fast-food and full-service restaurant consumption resulted in higher intakes of sugar $(3.95 \mathrm{~g}$ and $1.91 \mathrm{~g})$, saturated fat $(3.48 \mathrm{~g}$ and $2.52 \mathrm{~g})$ and $\mathrm{Na}(296.38 \mathrm{mg}$ and $451.06 \mathrm{mg}$ ).

Table 2 shows that the effects of fast-food restaurant consumption were significantly larger for young compared with older adults $(1138 \cdot 38 \mathrm{~kJ}(272 \cdot 25 \mathrm{kcal}) v .656 \cdot 16 \mathrm{~kJ}$

Table 1 Summary statistics for the full sample: respondents aged 20-64 years who completed $24 \mathrm{~h}$ dietary recall interviews for both day 1 and day 2 in the National Health and Nutrition Examination Survey (NHANES), 2003-2010

\begin{tabular}{|c|c|c|c|}
\hline Consumption and nutrient intake summary statistics & Day 1 & & Day 2 \\
\hline \multicolumn{4}{|l|}{ Prevalence of consumption } \\
\hline Fast-food restaurant (\%) & 35 & & 32 \\
\hline Fast-food restaurant away from home (\%) & 23 & & 21 \\
\hline Fast-food restaurant at home (\%) & 15 & & 13 \\
\hline Full-service restaurant (\%) & 28 & & 20 \\
\hline Full-service restaurant away from home (\%) & 23 & & 17 \\
\hline Full-service restaurant at home (\%) & 6 & & 4 \\
\hline Non-restaurant away from home (\%) & 61 & & 60 \\
\hline Consumption on weekday (\%) & 59 & & 80 \\
\hline \multicolumn{4}{|l|}{ Energy intake } \\
\hline \multicolumn{4}{|l|}{ Total energy (kJ) } \\
\hline Mean & 9548 & & 8866 \\
\hline SE & 54 & & 54 \\
\hline \multicolumn{4}{|l|}{ Total energy (kcal) } \\
\hline Mean & 2282 & & 2119 \\
\hline SE & 13 & & 13 \\
\hline \multicolumn{4}{|l|}{ Dietary nutrient intakes } \\
\hline \multicolumn{4}{|l|}{ Sugar $(\mathrm{g})$} \\
\hline Mean & 125 & & 116 \\
\hline SE & 1.0 & & 0.95 \\
\hline \multicolumn{4}{|l|}{ Saturated fat $(\mathrm{g})$} \\
\hline Mean & 29 & & 26 \\
\hline SE & 0.3 & & 0.3 \\
\hline \multicolumn{4}{|l|}{$\mathrm{Na}(\mathrm{mg})$} \\
\hline Mean & 3689 & & 3519 \\
\hline SE & $23 \cdot 8$ & & $21 \cdot 0$ \\
\hline Socio-economic statistics & \multicolumn{3}{|c|}{ Full sample } \\
\hline Male (\%) & & 48.90 & \\
\hline Young adults aged $20-34$ years (\%) & & 31.55 & \\
\hline Older adults aged $35-64$ years (\%) & & 68.45 & \\
\hline White (\%) & & 69.48 & \\
\hline Black (\%) & & 11.49 & \\
\hline Hispanic (\%) & & $13 \cdot 22$ & \\
\hline Other $(\%)$ & & $5 \cdot 81$ & \\
\hline Low income (\%) & & $18 \cdot 23$ & \\
\hline Middle income (\%) & & $25 \cdot 52$ & \\
\hline High income (\%) & & 51.03 & \\
\hline Full sample size & & 12528 & \\
\hline
\end{tabular}

All summary statistics are weighted using the NHANES examination weight. Low-income adults were defined as individuals in families with income $<130 \%$ of the federal poverty level (FPL), middle-income adults as $\geq 130 \%$ and $<300 \%$ of the FPL, and high-income adults as $\geq 300 \%$ of the FPL. 
Table 2 Regression estimates $(\beta)$ of the effect of fast-food and full-service restaurant consumption on energy and nutrient intakes by age and gender among respondents aged 20-64 years who completed $24 \mathrm{~h}$ dietary recall interviews for both day 1 and day 2 in the National Health and Nutrition Examination Survey (NHANES), 2003-2010

\begin{tabular}{|c|c|c|c|c|c|c|c|c|c|c|}
\hline & \multicolumn{2}{|c|}{ Energy (kJ) } & \multicolumn{2}{|c|}{ Energy (kcal) } & \multicolumn{2}{|c|}{ Sugar $(g)$} & \multicolumn{2}{|c|}{ Saturated fat $(g)$} & \multicolumn{2}{|c|}{$\mathrm{Na}(\mathrm{mg})$} \\
\hline & $\beta$ & SE & $\beta$ & SE & $\beta$ & SE & $\beta$ & SE & $\beta$ & SE \\
\hline \multicolumn{11}{|c|}{ Full sample ( $n 12$ 528) } \\
\hline Fast food & $813 \cdot 75^{\star \star *}$ & $73 \cdot 76$ & $194 \cdot 49^{\star \star *}$ & $17 \cdot 63$ & $3.95^{\star \star \star}$ & 1.39 & $3 \cdot 48^{\star * *}$ & 0.34 & $296 \cdot 38^{\star \star \star}$ & $36 \cdot 74$ \\
\hline Full service & $858 \cdot 04^{\star \star \star}$ & 88.59 & $205 \cdot 21^{\star \star \star}$ & $21 \cdot 17$ & 1.91 & 1.60 & $2.52^{\star \star \star}$ & 0.38 & $451 \cdot 06^{\star * *}$ & $45 \cdot 17$ \\
\hline \multicolumn{11}{|c|}{ Young adults aged $20-34$ years ( $n$ 3967) } \\
\hline Fast food & $1138 \cdot 38^{\star \star \star}$ & $129 \cdot 39$ & $272 \cdot 25^{\star \star *}$ & $30 \cdot 92$ & $6 \cdot 48^{\star \star \star}$ & 2.42 & $4 \cdot 69^{\star \star \star}$ & 0.56 & $374 \cdot 64^{\star * *}$ & $65 \cdot 22$ \\
\hline Full service & $981.96^{\star * *}$ & $155 \cdot 50$ & $234 \cdot 67^{\star \star *}$ & $37 \cdot 16$ & $6 \cdot 45^{\star *}$ & 3.05 & $2 \cdot 26^{\star \star \star}$ & 0.66 & $338 \cdot 29^{* * *}$ & $77 \cdot 73$ \\
\hline \multicolumn{11}{|c|}{ Older adults aged $35-64$ years ( $n$ 8561) } \\
\hline Fast food & $656 \cdot 16^{\star \star \star}$ & $89.64 \dagger$ & $156 \cdot 77^{\star \star \star}$ & $21.43 \dagger$ & $2 \cdot 90^{*}$ & 1.69 & $2 \cdot 87^{\star \star \star}$ & $0.42 \dagger$ & $255.09^{\star \star \star}$ & $44 \cdot 44$ \\
\hline Full service & $798.41^{\star \star \star}$ & $107 \cdot 77$ & $190 \cdot 99^{* \star *}$ & $25 \cdot 76$ & -0.26 & $1 \cdot 87 \dagger$ & $2 \cdot 66^{\star \star \star}$ & 0.47 & $503 \cdot 36^{\star \star *}$ & 55.43† \\
\hline \multicolumn{11}{|c|}{ Female ( $n$ 6424) } \\
\hline Fast food & $692 \cdot 49^{\star \star \star}$ & $83 \cdot 87$ & $165 \cdot 45^{\star \star \star}$ & $20 \cdot 05$ & $5 \cdot 93^{\star \star \star}$ & 1.63 & $2 \cdot 67^{\star \star \star}$ & 0.41 & $246 \cdot 73^{\star \star \star}$ & $41 \cdot 20$ \\
\hline Full service & $782 \cdot 31^{\star \star \star}$ & $97 \cdot 51$ & $187 \cdot 17^{\star \star *}$ & $23 \cdot 31$ & $3 \cdot 80^{* *}$ & 1.85 & $2 \cdot 18^{\star \star \star}$ & 0.45 & $388.56^{\star \star \star}$ & $51 \cdot 37$ \\
\hline \multicolumn{11}{|l|}{ Male ( $n$ 6104) } \\
\hline Fast food & $955 \cdot 60^{\star \star \star}$ & $120 \cdot 22 \dagger$ & $228 \cdot 47^{\star \star \star}$ & $28.73 \dagger$ & $2 \cdot 36$ & $2 \cdot 23$ & $4 \cdot 33^{\star \star \star}$ & $0.53 \dagger$ & $352 \cdot 10^{\star \star \star}$ & 60.58 \\
\hline Full service & $943 \cdot 37^{\star \star *}$ & 147.08 & $225 \cdot 52^{\star \star \star}$ & $35 \cdot 16$ & 0.12 & 2.59 & $2 \cdot 88^{\star \star \star}$ & 0.62 & $515 \cdot 17^{\star \star \star}$ & 73.96 \\
\hline
\end{tabular}

All analysis is weighted using the NHANES examination weight. Standard errors reported are robust. Control variable includes indicators for non-restaurant food away-from-home consumption, whether the recall was on a weekday $v$. a weekend day, and whether it was on day $1 v$. day 2 .

${ }^{*}$ Significant at $10 \%$ level, ${ }^{\star *}$ significant at $5 \%$ level, ${ }^{* \star *}$ significant at $1 \%$ level.

†Significant difference between young adults $v$. older adults, or female $v$. male, at the $P \leq 0.05$ level.

Table 3 Regression estimates $(\beta)$ of the effect of fast-food and full-service restaurant consumption on energy and nutrient intakes by race and income among respondents aged 20-64 years who completed $24 \mathrm{~h}$ dietary recall interviews for both day 1 and day 2 in the National Health and Nutrition Examination Survey (NHANES), 2003-2010

\begin{tabular}{|c|c|c|c|c|c|c|c|c|c|c|}
\hline & \multicolumn{2}{|c|}{ Energy (kJ) } & \multicolumn{2}{|c|}{ Energy (kcal) } & \multicolumn{2}{|c|}{ Sugar (g) } & \multicolumn{2}{|c|}{ Saturated fat $(\mathrm{g})$} & \multicolumn{2}{|c|}{$\mathrm{Na}(\mathrm{mg})$} \\
\hline & $\beta$ & SE & $\beta$ & SE & $\beta$ & SE & $\beta$ & SE & $\beta$ & $\mathrm{SE}$ \\
\hline \multicolumn{11}{|l|}{ White ( $n$ 5768) } \\
\hline Fast food & $715 \cdot 07^{\star \star \star}$ & 96.94 & $171.06^{\star \star *}$ & 23.17 & $3.03^{*}$ & 1.81 & $3 \cdot 13^{\star \star \star}$ & 0.45 & $244.99^{\star * \star}$ & 47.37 \\
\hline Full service & $909 \cdot 87^{\star \star \star}$ & $107 \cdot 17$ & $217 \cdot 58^{\star \star *}$ & $25 \cdot 61$ & 1.98 & 1.95 & $2 \cdot 73$ & $0.47^{\star \star \star}$ & 454.68 & $54 \cdot 03^{\star \star \star}$ \\
\hline \multicolumn{11}{|l|}{ Black (n 2682) } \\
\hline Fast food & $1241.49^{\star \star \star}$ & $154.60 \dagger$ & $296 \cdot 43^{\star \star \star}$ & $36.94 \dagger$ & $5 \cdot 71^{*}$ & 3.24 & $4 \cdot 74^{\star \star \star}$ & $0.64 \dagger$ & $451 \cdot 34^{\star \star \star}$ & $73.00 \dagger$ \\
\hline Full service & $1156 \cdot 69^{\star \star *}$ & 228.84 & $277 \cdot 11^{\star * *}$ & 54.70 & $13 \cdot 55^{\star \star \star}$ & $5.04 \dagger$ & $3.48^{\star \star \star}$ & 0.95 & $463.58^{\star \star \star}$ & $103 \cdot 87$ \\
\hline \multicolumn{11}{|c|}{ Hispanic (n 3517) } \\
\hline Fast food & $756 \cdot 82^{* \star \star}$ & $141.66 \S$ & $180 \cdot 83^{\star \star \star}$ & $33.86 \S$ & $7.57^{\star \star \star}$ & 2.34 & $3 \cdot 77^{\star \star \star}$ & 0.60 & $382 \cdot 91^{\star * \star}$ & $60 \cdot 63 \ddagger$ \\
\hline Full service & $755 \cdot 39^{\star \star \star}$ & 190.29 & $180 \cdot 68^{\star \star \star}$ & 45.48 & -0.37 & $3.13 \S$ & $2 \cdot 72^{\star \star \star}$ & 0.81 & $473 \cdot 93^{\star \star \star}$ & 94.52 \\
\hline \multicolumn{11}{|c|}{ Low income ( $n$ 3486) } \\
\hline Fast food & $945 \cdot 11^{\star \star \star}$ & $149 \cdot 94$ & $225 \cdot 87^{\star \star \star}$ & 35.84 & $6 \cdot 70^{\star *}$ & 2.94 & $3 \cdot 74^{\star \star \star}$ & 0.65 & $294.00^{\star \star \star}$ & $69 \cdot 45$ \\
\hline Full service & $773.92^{\star \star \star}$ & 226.88 & $185 \cdot 87^{\star \star \star}$ & 54.22 & 3.00 & $4 \cdot 27$ & $1 \cdot 18$ & 0.91 & $324 \cdot 17^{\star \star \star}$ & 110.47 \\
\hline \multicolumn{11}{|c|}{ Middle income ( $n$ 3464) } \\
\hline Fast food & $1002 \cdot 47^{\star \star \star}$ & $140 \cdot 86$ & $239 \cdot 61^{\star \star \star}$ & 33.66 & $7 \cdot 48^{\star \star \star}$ & 2.65 & $4 \cdot 20^{\star \star \star}$ & 0.63 & $388 \cdot 30^{\star \star \star}$ & $70 \cdot 84$ \\
\hline Full service & $1069 \cdot 31^{\star \star \star}$ & 178.58 & $255.48^{\star \star \star}$ & $42 \cdot 68$ & 4.84 & 3.28 & $3 \cdot 04^{\star \star \star}$ & 0.79 & $514 \cdot 27^{\star \star \star}$ & $88 \cdot 13$ \\
\hline \multicolumn{11}{|c|}{ High income ( $n$ 4747) } \\
\hline Fast food & $653 \cdot 17^{\star \star \star}$ & $109 \cdot 81 \S$ & $156 \cdot 10^{\star \star *}$ & $26 \cdot 25 \S$ & 1.37 & $2.00 \S$ & $3 \cdot 10^{\star \star \star}$ & 0.51 & $233 \cdot 10^{* * *}$ & $55 \cdot 68 \S$ \\
\hline Full service & $804 \cdot 77^{\star \star \star}$ & 118.55 & $192 \cdot 47^{\star \star \star}$ & $28 \cdot 34$ & 0.05 & $2 \cdot 12$ & $2 \cdot 56^{\star \star \star}$ & 0.51 & $463 \cdot 39^{\star \star \star}$ & 61.65 \\
\hline
\end{tabular}

All analysis is weighted using the NHANES examination weight. Standard errors reported are robust. Control variable includes indicators for non-restaurant food away-from-home consumption, whether the recall was on a weekday $v$. a weekend day, and whether it was on day $1 v$. day 2 . Some subgroup observation counts do not equal the total of 12528 because: (i) results were not reported for all race/ethnicity subgroups such as Asian and other races; and (ii) data on income were missing for some observations.

${ }^{*}$ Significant at $10 \%$ level, ${ }^{* *}$ significant at $5 \%$ level, ${ }^{* \star *}$ significant at $1 \%$ level.

†Significant difference between white $v$. black, or low income $v$. middle income, at the $P \leq 0.05$ level.

$\ddagger$ Significant difference between white $v$. Hispanic, or low income $v$. high income, at the $P \leq 0.05$ level.

$\S$ Significant difference between black $v$. Hispanic, or middle income $v$. high income, at the $P \leq 0.05$ level.

$(156 \cdot 77 \mathrm{kcal}))$ and for males compared with females (955.60 kJ (228.47 kcal) v. $692.49 \mathrm{~kJ}(165.45 \mathrm{kcal}))$. Young adult and male fast-food restaurant customers also consumed more additional saturated fat than their respective counterparts. There were no statistically significant differences by age or gender in the effects of full-service restaurant consumption (except for the effect on sugar intake which was greater among young $v$. older adults).

Table 3 shows that there were a number of significant differences found by race/ethnicity and income 
sub-populations. Fast-food restaurant consumption was associated with greater additional energy intake for blacks (1241.49 kJ (296.43 kcal)) compared with whites $(715.07 \mathrm{~kJ}$ (171.06 kcal)) and Hispanics $(756.82 \mathrm{~kJ}$ (180.83 kcal)). In addition, fast-food consumption was associated with higher additional saturated fat and $\mathrm{Na}$ intakes for black ( $4.74 \mathrm{~g}$ and $451.34 \mathrm{mg}$, respectively) compared with white (3.13 g and $244.99 \mathrm{mg}$, respectively) adults and higher $\mathrm{Na}$ intake compared with Hispanic adults $(382.91 \mathrm{mg})$. Fastfood restaurant consumption had the least adverse impacts on higher-income adults' diets. Specifically, consuming fast food had virtually no impact on high-income adults' sugar intake, and a smaller adverse impact on their total energy intake compared with their middle-income counterparts $(653.17 \mathrm{~kJ}(156 \cdot 10 \mathrm{kcal}) v .1002 .47 \mathrm{~kJ}(239.61 \mathrm{kcal}))$. Consuming fast food also was associated with a lower increase in $\mathrm{Na}$ intake among high-income compared with middle-income adults $(233.10 \mathrm{mg} v .388 .30 \mathrm{mg})$. Similar to the findings by age and gender, there were generally no significant differences in the effect of full-service restaurant consumption by race/ethnicity or income (with the exception of differences in sugar intake by race/ethnicity).

In Table 4, we further explore the differences in total energy intake by race/ethnicity and income across age and gender. For young adults, restaurant food consumption, despite its adverse effects, had virtually no differential effects among racial and income subgroups except that Hispanics had lower additional total energy intake than black adults when eating at a fast-food restaurant. In contrast, older adult, males and females exhibited substantial differential effects of consuming fast food across racial and income subgroups. Fast-food consumption was associated with higher additional energy intake for black compared with white adults, regardless of age or gender. For example, consumption of fast food was associated with an additional $1345.18 \mathrm{~kJ}(321.51 \mathrm{kcal})$ among black male adults compared with $851.13 \mathrm{~kJ}(203.42 \mathrm{kcal})$ among white male adults. Within black adults, however, we did not observe differences in additional energy intake across age and gender groups.

As illustrated in Table 4, fast-food consumption was associated with the highest additional energy intake for middle-income males $(1457.50 \mathrm{~kJ}(348.35 \mathrm{kcal}))$ compared with high-income $(763.99 \mathrm{~kJ}(182.60 \mathrm{kcal}))$ and low-income males $(782.48 \mathrm{~kJ}(187.02 \mathrm{kcal}))$. However, fast-food restaurant consumption was associated with the highest additional energy intake among low-income females (1061.28 kJ (253.65 kcal)) compared with their middle-income $(538.63 \mathrm{~kJ}$ (128.74 kcal)) and high-income $(565.38 \mathrm{~kJ}(135.13 \mathrm{kcal}))$ counterparts. Fast-food consumption also was associated with lower additional energy intake for high-income older adults compared with their middle-income counterparts $(550.48 \mathrm{~kJ}(131.57 \mathrm{kcal}) v .922 .46 \mathrm{~kJ}(220.47 \mathrm{kcal}))$.

In Table 5, we show the results of the effects of fast-food and full-service restaurant consumption by meal occasion. Fast-food consumption as a snack was associated with the highest additional energy intake (1091.98 kJ (260.99 kcal)) followed by fast-food consumption for dinner $(818.57 \mathrm{~kJ}$ (195.64 kcal)) and lunch $(701.41 \mathrm{~kJ}(167.64 \mathrm{kcal}))$. Fastfood consumption for breakfast was associated with the least additional energy intake $(354.44 \mathrm{~kJ}(84.71 \mathrm{kcal}))$. Similarly, full-service restaurant consumption as a snack was associated with the highest additional energy intake $(979.91 \mathrm{~kJ}(234.20 \mathrm{kcal}))$ followed by consumption for dinner $(903.02 \mathrm{~kJ} \quad(215.83 \mathrm{kcal}))$, breakfast $(611.21 \mathrm{~kJ}$ (146.08 kcal)) and lunch (480.74 kJ (114.90 kcal)).

\section{Discussion and conclusion}

Based on multivariate individual-level fixed-effects models, the present study shows that both fast-food and full-service restaurant food consumption among adults were associated with significant increases in energy, sugar, saturated fat and $\mathrm{Na}$ intakes. Previous research suggested that on days that adults consume from restaurant sources, they consumed $2410 \mathrm{~kJ}(576 \mathrm{kcal})$ from fast-food restaurants $(31 \%$ of total energy intake) and $2858 \mathrm{~kJ}(683 \mathrm{kcal})$ from full-service restaurants $(34 \% \text { of total energy intake })^{(4)}$. In our research, restaurant consumption was associated with an additional total daily energy intake of $813.75 \mathrm{~kJ}(194.49 \mathrm{kcal})$ for fastfood and $858.04 \mathrm{~kJ}(205.21 \mathrm{kcal})$ for full-service restaurants. Restaurant food consumption as a snack was associated with particularly high additional energy intake (1091.98 kJ $(260.99 \mathrm{kcal})$ for fast-food and $979.91 \mathrm{~kJ}(234.20 \mathrm{kcal})$ for full-service restaurants). Therefore, the evidence suggests that adults do not sufficiently reduce non-restaurant intake to compensate for additional energy intake on days consuming at restaurants. Further, in terms of nutrient intakes, both fast-food and full-service restaurant consumption increased intakes of saturated fat $(3.48 \mathrm{~g}$ and $2.52 \mathrm{~g}$, respectively), sugar (3.95 g and $1.91 \mathrm{~g}$, respectively) and $\mathrm{Na}$ (296.38 $\mathrm{mg}$ and $451.06 \mathrm{mg}$, respectively).

Our results are consistent with previous studies that examined adult restaurant food consumption. Evidence from earlier studies based on cross-sectional analysis and mean comparisons using the Continuing Survey of Food Intakes by Individuals (CSFII) 1994-1996 showed that adults who reported eating fast food had higher intakes of energy, total fat, saturated fat, $\mathrm{Na}$ and carbonated soft drinks, and lower intakes of vitamin A, vitamin C, Ca, milk, fruits and vegetables than those who did not report eating fast food ${ }^{(8,9)}$, and that meals consumed at both fast-food and full-service restaurants were associated with higher energy intake ${ }^{(10)}$. Assessing FAFH, two studies using withinperson estimation based on NHANES 2003-2004 data and the CSFII data found that FAFH increased adults' daily energy intake and reduced diet quality from a classification of fair to poor ${ }^{(7,12)}$. While the conclusion is similar, our study with more recent waves of NHANES data and explicit specifications of fast-food and full-service restaurant consumption found larger adverse effects. 
Table 4 Regression estimates $(\beta)$ of the effect of fast-food and full-service restaurant consumption on energy intake by gender, race and socio-economic status for young (aged 20-34 years) and older (aged 35-64 years) adults, and male and female adults, among respondents aged 20-64 years who completed $24 \mathrm{~h}$ dietary recall interviews for both day 1 and day 2 in the National Health and Nutrition Examination Survey (NHANES), 2003-2010

\begin{tabular}{|c|c|c|c|c|c|c|c|c|}
\hline \multirow[b]{2}{*}{ Energy (kJ) } & \multicolumn{2}{|c|}{ Young adult } & \multicolumn{2}{|c|}{ Older adult } & \multicolumn{2}{|c|}{ Male } & \multicolumn{2}{|c|}{ Female } \\
\hline & $\beta$ & $\mathrm{SE}$ & $\beta$ & $\mathrm{SE}$ & $\beta$ & $\mathrm{SE}$ & $\beta$ & $\mathrm{SE}$ \\
\hline \multicolumn{9}{|l|}{ White $(n 5768)$} \\
\hline Fast food & $1112 \cdot 02^{\star \star *}$ & $180 \cdot 73$ & $548 \cdot 43^{\star \star \star}$ & $114.45 \dagger$ & $851 \cdot 13^{\star \star \star}$ & $156 \cdot 00$ & $602 \cdot 39^{\star \star \star}$ & 110.33† \\
\hline Full service & $951.04^{\star \star \star}$ & $199 \cdot 10$ & $886 \cdot 43^{\star \star \star}$ & 127.03 & $1014 \cdot 44^{\star \star \star}$ & 176.95 & $810 \cdot 83^{\star \star \star}$ & $119 \cdot 41$ \\
\hline \multicolumn{9}{|l|}{ Black ( $n$ 2682) } \\
\hline Fast food & $1376 \cdot 52^{\star \star \star}$ & 285.75 & $1157 \cdot 67^{\star \star \star}$ & $176.59 \ddagger$ & $1345 \cdot 18^{\star \star \star}$ & $259.85 \ddagger$ & $1160 \cdot 27^{\star \star *}$ & $186 \cdot 91 \ddagger$ \\
\hline Full service & $1641 \cdot 42^{\star \star \star}$ & $412 \cdot 41$ & $829 \cdot 35^{\star \star \star}$ & $264.80 \dagger$ & $1630 \cdot 11^{\star * *}$ & 370.12 & $698 \cdot 16^{\star \star}$ & $271.74 \dagger$ \\
\hline \multicolumn{9}{|c|}{ Hispanic ( $n$ 3517) } \\
\hline Fast food & $784 \cdot 42^{\star \star \star}$ & $212 \cdot 01 \S$ & $756 \cdot 89^{\star \star \star}$ & $189 \cdot 32$ & $1032 \cdot 09^{\star \star \star}$ & $229 \cdot 07$ & $475 \cdot 82^{\star \star \star}$ & $162 \cdot 86 \S, \dagger$ \\
\hline Full service & $937 \cdot 64^{\star \star \star}$ & 298.35 & $608 \cdot 45^{\star \star}$ & 245.01 & $698 \cdot 03^{\star \star}$ & $323.62 \S$ & $825 \cdot 26^{\star \star \star}$ & $194 \cdot 32$ \\
\hline \multicolumn{9}{|c|}{ Low income ( $n$ 3486) } \\
\hline Fast food & $1283 \cdot 71^{\star \star \star}$ & 230.04 & $707 \cdot 78^{\star \star \star}$ & $195.92 \dagger$ & $782 \cdot 48^{\star \star \star}$ & 268.28 & $1061 \cdot 28^{\star \star \star}$ & $164 \cdot 78$ \\
\hline Full service & $1183 \cdot 76^{\star * *}$ & 356.48 & 454.90 & 286.01 & $775 \cdot 00^{\star *}$ & $392 \cdot 14$ & $778.91^{* * *}$ & 244.03 \\
\hline \multicolumn{9}{|c|}{ Middle income ( $n$ 3464) } \\
\hline Fast food & $1114 \cdot 69^{\star \star \star}$ & $238 \cdot 14$ & $922 \cdot 46^{\star \star \star}$ & 171.47 & $1457 \cdot 50^{\star \star \star}$ & $219 \cdot 83 \ddagger$ & $538 \cdot 63^{\star \star *}$ & $168 \cdot 28 \ddagger, \dagger$ \\
\hline Full service & $989 \cdot 17^{\star \star \star}$ & 292.85 & $1104 \cdot 76^{\star \star \star}$ & $226 \cdot 22 \ddagger$ & $937 \cdot 77^{\star \star \star}$ & 294.06 & $1163 \cdot 35^{\star \star \star}$ & 203.45 \\
\hline \multicolumn{9}{|c|}{ High income (n 4747) } \\
\hline Fast food & $972 \cdot 10^{\star \star \star}$ & $219 \cdot 66$ & $550 \cdot 48^{\star \star \star}$ & $126 \cdot 84 \S, \dagger$ & $763.99^{\star \star \star}$ & $176 \cdot 30 \S$ & $565 \cdot 38^{\star \star \star}$ & $124 \cdot 59 \|$ \\
\hline \multirow[t]{2}{*}{ Full service } & $925 \cdot 59^{\star * \star}$ & $227 \cdot 79$ & $758.00^{\star * *}$ & 138.94 & $988 \cdot 37^{\star * \star}$ & $195 \cdot 38$ & $630 \cdot 13^{\star \star *}$ & $128 \cdot 19 \S$ \\
\hline & \multicolumn{2}{|c|}{ Young adult } & \multicolumn{2}{|c|}{ Older adult } & \multicolumn{2}{|c|}{ Male } & \multicolumn{2}{|c|}{ Female } \\
\hline Energy (kcal) & $\beta$ & SE & $\beta$ & SE & $\beta$ & SE & $\beta$ & SE \\
\hline \multicolumn{9}{|l|}{ White ( $n$ 5768) } \\
\hline Fast food & $265 \cdot 78^{\star \star \star}$ & $43 \cdot 19$ & $131 \cdot 08^{\star \star \star}$ & $27 \cdot 35 \dagger$ & $203 \cdot 42^{\star \star \star}$ & 37.28 & $143 \cdot 97^{\star \star \star}$ & $26 \cdot 37 \dagger$ \\
\hline Full service & $227 \cdot 30^{\star \star \star}$ & 47.59 & $211 \cdot 86^{\star \star \star}$ & $30 \cdot 36$ & $242 \cdot 46^{\star \star \star}$ & $42 \cdot 29$ & $193 \cdot 79^{\star \star \star}$ & 28.54 \\
\hline \multicolumn{9}{|l|}{ Black ( $n$ 2682) } \\
\hline Fast food & $329 \cdot 00^{\star \star \star}$ & $68 \cdot 30$ & $276 \cdot 69^{\star \star \star}$ & $42 \cdot 21 \ddagger$ & $321.51^{\star \star \star}$ & $62 \cdot 11 \ddagger$ & $277 \cdot 31^{\star \star \star}$ & $44 \cdot 67 \ddagger$ \\
\hline Full service & $392 \cdot 31^{\star \star \star}$ & 98.57 & $198 \cdot 22^{\star \star \star}$ & $63.29 \dagger$ & $389.61^{\star \star \star}$ & 88.46 & $166 \cdot 86^{\star \star}$ & $64.95 \dagger$ \\
\hline \multicolumn{9}{|c|}{ Hispanic ( $n$ 3517) } \\
\hline Fast food & $187 \cdot 48^{\star \star \star}$ & $50 \cdot 67 \S$ & $180 \cdot 90^{\star \star \star}$ & $45 \cdot 25$ & $246 \cdot 68^{\star \star \star}$ & 54.75 & $113 \cdot 72^{\star \star \star}$ & $38.92 \S, \uparrow$ \\
\hline Full service & $224 \cdot 10^{\star \star \star}$ & $71 \cdot 31$ & $145 \cdot 42^{\star \star}$ & 58.56 & $166 \cdot 83^{\star *}$ & $77.35 \S$ & $197 \cdot 24^{\star \star \star}$ & 46.44 \\
\hline \multicolumn{9}{|c|}{ Low income ( $n$ 3486) } \\
\hline Fast food & $306 \cdot 81^{\star \star \star}$ & 54.98 & $169 \cdot 16^{\star \star \star}$ & $46 \cdot 83 \dagger$ & $187 \cdot 02^{\star \star \star}$ & $64 \cdot 12$ & $253.65^{\star \star \star}$ & 39.38 \\
\hline Full service & $282 \cdot 92^{\star \star \star}$ & $85 \cdot 20$ & $108 \cdot 72$ & 68.36 & $185 \cdot 23^{\star *}$ & $93 \cdot 72$ & $186 \cdot 16^{\star * \star}$ & 58.32 \\
\hline \multicolumn{9}{|c|}{ Middle income ( $n$ 3464) } \\
\hline Fast food & $266 \cdot 42^{\star \star \star}$ & $56 \cdot 92$ & $220 \cdot 47^{\star \star \star}$ & $40 \cdot 98$ & $348 \cdot 35^{\star \star *}$ & $52 \cdot 54 \ddagger$ & $128 \cdot 74^{\star * \star}$ & $40 \cdot 22 \ddagger, \uparrow$ \\
\hline Full service & $236 \cdot 42^{\star \star \star}$ & 69.99 & $264 \cdot 04^{\star \star \star}$ & $54.07 \ddagger$ & $224 \cdot 13^{\star \star \star}$ & $70 \cdot 28$ & $278.05^{\star \star \star}$ & 48.63 \\
\hline \multicolumn{9}{|c|}{ High income ( $n$ 4747) } \\
\hline Fast food & $232 \cdot 34^{\star \star \star}$ & 52.50 & $131.57^{\star \star \star}$ & $30 \cdot 32 \S, \dagger$ & $182 \cdot 60^{\star \star \star}$ & $42 \cdot 14 \S$ & $135 \cdot 13^{\star \star \star}$ & $29 \cdot 78 \|$ \\
\hline Full service & $221 \cdot 22^{\star \star \star}$ & 54.44 & $181 \cdot 17^{\star \star \star}$ & 33.21 & $236 \cdot 23^{\star \star \star}$ & $46 \cdot 70^{\circ}$ & $150 \cdot 60^{\star \star \star}$ & $30.64 \S$ \\
\hline
\end{tabular}

All analysis is weighted using NHANES examination weight. Standard errors reported are robust. Control variable includes indicators for non-restaurant food away-from-home consumption, whether the recall was on a weekday $v$. a weekend day, and whether it was on day $1 v$. day 2 . Some subgroup observation counts do not equal the total of 12528 because: (i) results were not reported for all race/ethnicity subgroups such as Asian and other races; and (ii) data on income were missing for some observations.

** Significant at $5 \%$ level, ${ }^{* * *}$ significant at $1 \%$ level.

†Significant difference between young adults $v$. older adults, or female $v$. male, at the $P \leq 0.05$ level.

$\ddagger$ Significant difference between white $v$. black, or low income $v$. middle income, at the $P \leq 0.05$ level.

$\S$ Significant difference between black $v$. Hispanic, or middle income $v$. high income, at the $P \leq 0.05$ level.

ISSignificant difference between white $v$. Hispanic, or low income $v$. high income, at the $P \leq 0.05$ level.

The present study provides new evidence for adults on the differential effects across racial and income subgroups. Similar to a recent study for children ${ }^{(13)}$, we found that fastfood consumption was associated with fewer additional total kilojoules and lower sugar and $\mathrm{Na}$ intakes for high-income adults. Further, fast-food consumption was associated with greater additional energy, saturated fat and $\mathrm{Na}$ intakes for black compared with white adults, which was similarly found in adolescents ${ }^{(13)}$. Overall, individual characteristics moderated the impacts of restaurant food consumption on adults' diets. The study also documents the racial and income differences between young $v$. older adults, and for male $v$. female adults. Fast-food consumption resulted in lower additional energy intake for high-income older adults (compared with middle-income older adults). By gender subgroup, middle-income male adults and low-income female adults were the two groups with the highest additional energy intake associated with fast-food consumption.

The results suggest that there were differences across racial and income groups by gender and age on energy 
Table 5 Regression estimates $(\beta)$ of the effect of fast-food and full-service restaurant consumption on energy intake by meal occasion among respondents aged 20-64 years who completed $24 \mathrm{~h}$ dietary recall interviews for both day 1 and day 2 in the National Health and Nutrition Examination Survey (NHANES), 2003-2010

\begin{tabular}{|c|c|c|c|c|}
\hline \multirow[b]{2}{*}{ Sample (n 12 528) } & \multicolumn{2}{|c|}{ Energy (kJ) } & \multicolumn{2}{|c|}{ Energy (kcal) } \\
\hline & $\beta$ & SE & $\beta$ & SE \\
\hline Fast food: Breakfast & $354.44^{\star \star \star}$ & 0.57 & $84 \cdot 71^{\star \star \star}$ & 0.14 \\
\hline Fast food: Lunch & $701.41^{\star \star \star}$ & $0.36 \dagger$ & $167 \cdot 64^{\star \star \star}$ & $0.09+$ \\
\hline Fast food: Dinner & $818.57^{\star \star *}$ & $0.37 \ddagger, \S$ & $195.64^{* \star *}$ & $0.09 \ddagger, \S$ \\
\hline Fast food: Snack & $1091.98^{\star \star \star}$ & $0.59 \|, \boldsymbol{\eta}, \dagger \dagger$ & $260 \cdot 99^{\star \star \star}$ & $0.14 \|, \boldsymbol{\eta}, \dagger \dagger$ \\
\hline Full service: Breakfast & $611 \cdot 21^{\star \star \star}$ & 0.71 & $146 \cdot 08^{\star \star \star}$ & 0.17 \\
\hline Full service: Lunch & $480 \cdot 74^{\star \star *}$ & $0.45 \dagger$ & $114 \cdot 90^{\star \star \star}$ & $0.11 \dagger$ \\
\hline Full service: Dinner & $903.02^{\star \star \star}$ & $0.40 \ddagger, \S$ & $215 \cdot 83^{\star \star \star}$ & $0.10 \ddagger, \S$ \\
\hline Full service: Snack & $979 \cdot 91^{\star \star *}$ & $0.711, \boldsymbol{\eta}$, ,† & $234 \cdot 20^{\star \star \star}$ & $0.17 \|, \mathbb{\Pi},+\dagger$ \\
\hline
\end{tabular}

All analysis is weighted using NHANES examination weight. Standard errors reported are robust. Control variable includes indicators for non-restaurant food away-from-home consumption, whether the recall was on a weekday $v$. a weekend day, and whether it was on day $1 v$. day 2 . *** Significant at $1 \%$ level.

†Significant difference between breakfast $v$. lunch at the $P \leq 0.05$ level

$¥$ Significant difference between dinner $v$. lunch at the $P \leq 0.05$ level.

$\S$ Significant difference dinner $v$. breakfast at the $P \leq 0.05$ level.

IISignificant difference between snack $v$. dinner at the $P \leq 0.05$ level.

TSignificant difference between snack $v$. lunch at the $P \leq 0.05$ level.

t†Significant difference between snack $v$. breakfast at the $P \leq 0.05$ level.

intake and diet outcomes, which may lead to potential health disparities. Fast-food restaurant consumption had a consistently greater adverse effect on energy intake among black adults compared with white and Hispanic adults, for older adults and both male and female adults. This finding that blacks have greater adverse effects from consuming fast food is even more concerning given other evidence that they consume a higher percentage of their total energy from fast-food restaurants than their white and Hispanic counterparts ${ }^{(22)}$.

There are several limitations to the present study. First, the data were self-reported based on $24 \mathrm{~h}$ dietary recalls. Validation studies have reported the tendency towards overestimation of portion size by those who eat smaller portions and underestimation by those who eat larger portions ${ }^{(23)}$. A study that used NHANES III suggested that $28 \%$ of the women and $18 \%$ of the men were classified as under-reporters, with under-reporting of energy intake highest in individuals who were older, overweight or trying to lose weight ${ }^{(24,25)}$. Second, our individual fixedeffects model was able to account for some but not all of the bias and under-report issues. Third, due to data limitations, the regression analyses could not incorporate the level of physical activity on the interview day or other unobserved time-varying factors that might affect food preferences and food demand day to day. Despite these limitations, the fixed-effects regression estimation was able to control for individuals' unobserved time-constant characteristics and preferences.

There is a rising interest in potential FAFH policy interventions to alleviate factors related to the obesity epidemic, including prohibitions on new fast-food restaurants' opening, increasing the relative costs of restaurant food purchases, and mandatory posting of energy (calories) on menus in chain restaurants ${ }^{(26,27)}$. Consistent with previous study findings for children and adolescents, our research results for adults suggest that given that full-service and fastfood restaurant consumption were similarly related to excess energy intake and a number of poorer dietary outcomes, policies intended to limit access to unhealthy foods and beverages should apply to both full-service and fastfood restaurants ${ }^{(13)}$. In addition, given that larger adverse effects on energy intake and diet quality were found for some lower socio-economic and minority populations, policies aimed at improving diet and reducing energy intake from restaurant sources will help to reduce racial and socio-economic disparities in Americans' diets.

\section{Acknowledgements}

Financial support: This research was supported by the National Heart, Lung, and Blood Institute (grant number R01HL096664). The content is solely the responsibility of the authors and does not necessarily represent the official views of the National Heart, Lung, and Blood Institute or the National Institutes of Health. The funders had no role in the design, analysis or writing of this article. Conflict of interest: None. Authorship: study concept and design, B.T.N and L.M.P.; acquisition of data, L.M.P.; analysis and interpretation of data, B.T.N and L.M.P.; drafting of the manuscript, B.T.N.; critical revision of the manuscript for important intellectual content, B.T.N and L.M.P.; statistical analysis, B.T.N and L.M.P.; obtained funding, L.M.P. Ethics of buman subject participation: The study was approved by the Institutional Review Board of the University of Illinois at Chicago.

\section{References}

1. Flegal KM, Carroll MD, Kit BK et al. (2012) Prevalence of obesity and trends in the distribution of body mass index among US adults, 1999-2010. JAMA 307, 491-497. 
2. Bray GA (2004) Medical consequences of obesity. J Clin Endocrinol Metab 89, 2583-2589.

3. Finkelstein EA, Trogdon JG, Cohen JW et al. (2009) Annual medical spending attributable to obesity: payer- and servicespecific estimates. Health Aff (Millwood) 28, w822-w831.

4. Powell LM, Nguyen BT \& Han E (2012) Energy intake from restaurants: demographics and socioeconomics, 2003-2008. Am J Prev Med 43, 498-504.

5. Nielsen SJ, Siega-Riz AM \& Popkin BM (2002) Trends in energy intake in US between 1977 and 1996: similar shifts seen across age groups. Obes Res 10, 370-378.

6. US Department of Agriculture, Economic Research Service (2013) Food Expenditure Briefing Room - Table 10. http:// www.ers.usda.gov/data-products/food-expenditures.aspx\#. U483ePldUSU (accessed April 2014).

7. Mancino L, Todd J \& Lin B-H (2009) Separating what we eat from where: measuring the effect of food away from home on diet quality. Food Policy 34, 557-562.

8. Paeratakul S, Ferdinand DP, Champagne CM et al. (2003) Fast-food consumption among US adults and children: dietary and nutrient intake profile. J Am Diet Assoc 103, 1332-1338.

9. Bowman SA \& Vinyard BT (2004) Fast food consumption of US adults: impact on energy and nutrient intakes and overweight status. J Am Coll Nutr 23, 163-168.

10. Binkley JK (2008) Calorie and gram differences between meals at fast food and table service restaurants. Rev Agric Econ 30, 750-763.

11. Larson N, Neumark-Sztainer D, Laska MN et al. (2011) Young adults and eating away from home: associations with dietary intake patterns and weight status differ by choice of restaurant. J Am Diet Assoc 111, 1696-1703.

12. Todd JE, Mancino L \& Lin B (2010) The Impact of Food Away From Home on Adult Diet Quality. Economic Research Report no. ERR-90. Washington, DC: USDA/ERS.

13. Powell LM \& Nguyen BT (2013) Fast-food and full-service restaurant consumption among children and adolescents: effect on energy, beverage, and nutrient intake. JAMA Pediatr 167, 14-20.

14. Flegal KM, Carroll MD, Ogden CL et al. (2012) Prevalence and trends in obesity among US adults, 1999-2008. JAMA 303, 235-241.

15. Mancino L, Todd JE, Guthrie J et al. (2010) How Food Away From Home Affects Children's Diet Quality.
Economic Research Report no. ERR-104. Washington, DC: USDA/ERS.

16. Johnson RK, Appel LJ, Brands M et al. (2009) Dietary sugars intake and cardiovascular health: a scientific statement from the American Heart Association. Circulation 120, 1011-1020.

17. Bibbins-Domingo K, Chertow GM, Coxson PG et al. (2010) Projected effect of dietary salt reductions on future cardiovascular disease. $N$ Engl J Med 362, 590-599.

18. Eichtenstein AH, Kennedy E, Barrier P et al. (1998) Dietary fat consumption and health. Nutr Rev 56, 3-19.

19. Centers for Disease Control and Prevention, National Center for Health Statistics (2013) National Health and Nutrition Examination Survey. http://www.cdc.gov/nchs/nhanes.htm (accessed May 2013).

20. Centers for Disease Control and Prevention (2008) National Health and Nutrition Examination Survey (NHANES): MEC in-person dietary interviewers procedures manual. http://www.cdc.gov/nchs/data/nhanes/nhanes_07_08/manual_ dietarymec.pdf (accessed November 2013).

21. Haines PS, Hama MY, Guilkey DK et al. (2003) Weekend eating in the United States is linked with greater energy, fat, and alcohol intake. Obes Res 11, 945-949.

22. Fryar CD \& Ervin RB (2013) Caloric Intake From Fast Food Among Adults: United States, 2007-2010. NCHS Data Brief no. 114. Hyattsville, MD: National Center for Health Statistics.

23. Faggiano F, Vineis P, Cravanzola D et al. (1992) Validation of a method for the estimation of food portion size. Epidemiology 3, 379-382.

24. Mertz W, Tsui JC, Judd J et al. (1991) What are people really eating? The relation between energy intake derived from estimated diet records and intake determined to maintain body weight. Am J Clin Nutr 54, 291-295.

25. Briefel RR, Sempos CT, McDewell MA et al. (1997) Dietary methods research in the Third National Health and Nutrition Examination Survey: underreporting of energy intake. Am J Clin Nutr 65, 4 Suppl, 1203S-1209S.

26. Sturm R \& Cohen DA (2009) Zoning for health? The year-old ban on new fast-food restaurants in South LA. Health Aff (Millwood) 28, w1088-w1097.

27. Bollinger B, Leslie P \& Sorensen A (2011) Calorie posting in chain restaurants. Am Econ J Econ Policy 3, 91-128. 